\title{
Forgetting to forget: On the duration of voluntary suppression of neutral and emotional memories
}

\author{
Simon Nørby, Martin Lange \& Axel Larsen (2010)
}

Using Anderson and Green's (2001) "think/no-think" paradigm with neutral and emotional nouns, we found in agreement with other studies that memory for neutral words was reduced instantly upon repeated attempts at suppression. However, the effect was temporary and vanished after a period of one week, which suggests that memory suppression interferes with immediate retrieval but does not lead to long-term forgetting. Furthermore, the amount of training that reduced immediate recall of neutral items did not at all reduce recall of emotional items. This finding is in accordance with the notion that emotional items have a higher degree of salience and tend to attract more attention than neutral items.

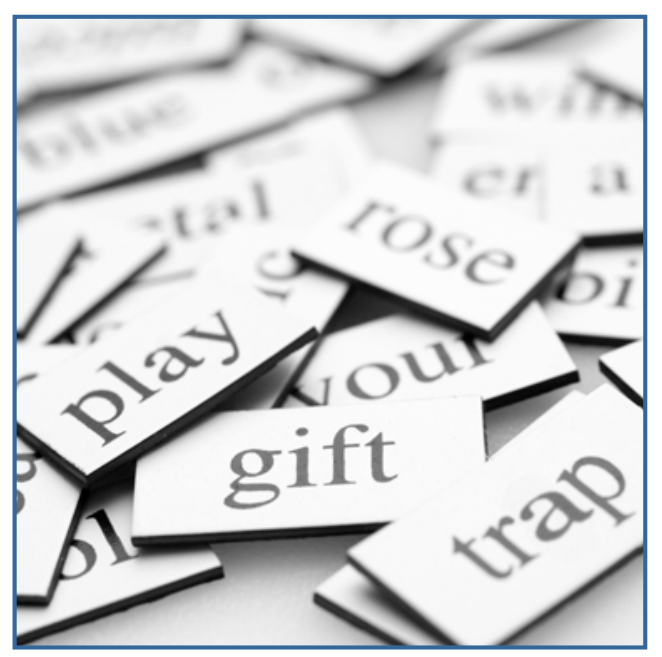

Background

In the think/no-think (TNT) paradigm, participants are to avoid that certain memories enter their minds. First, they learn a number of cue-target pairs. Then, in the critical phase, they are continuously exposed to cues and instructed to suppress (not think about) the associated targets in relation to some of these cues while rehearsing (think about) the targets for the rest of them. When tested later on, recall of items attempted suppressed are typically inferior to recall of studied baseline targets not involved in the critical phase of the experiment (e.g., Anderson \& Green, 2001; Anderson et al., 2004).

The present study investigated suppression of neutral and emotional targets and tested the "long-term" effect of suppression. Emotional material (such as emotional words) is typically remembered better than neutral material (see Reisberg \& Heuer, 2004). Therefore, it was hypothesized that emotional targets would be harder to suppress than neutral targets. To test the "long-term" effect of suppression, we employed a retest after one week.

\section{Method}

Stimuli comprised 35 neutral and 35 emotional cuetarget word pairs (all words were nouns). A neutral word pair consisted of two neutral words (e.g., "habitbalcony"). An emotional word pair consisted of a neutral cue word and an emotional target word (e.g., "jungle-incest").

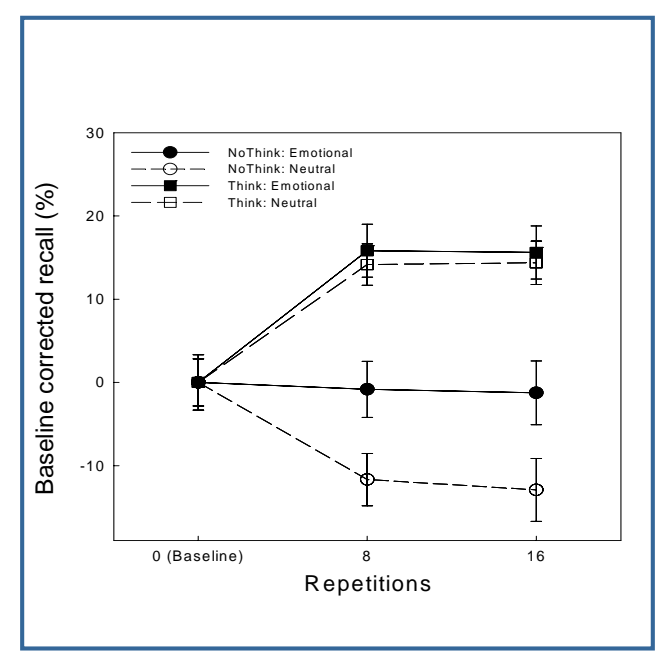

In the study phase, the subject was exposed twice to all the word pairs. In the training phase, the subject was shown cues one by one, and asked to recall the associated targets. Test-feedback cycles continued until accuracy was at least $60 \%$.

Cues from 60 randomly assigned word pairs were presented in the TNT phase while cues from the remaining 10 baseline word pairs were not shown in this phase. The participant memorized (accuracy $90 \%$ ) a subset of 20 cues in which the target was to be suppressed later on. The 60 cues were then shown on 8 or 16 occasions and should either be responded or not responded to (respectively rehearsed or suppressed)

In the test phase, the individual was asked to report the target to each of the 70 cues irrespective of prior instructions. After completing this phase, he or she was asked to return after one week in order to participate in a seemingly unrelated experiment. The re-test was similar to the test phase.

\section{Results}

The left figure show immediate recall as a function of repetitions with valence and TNT instructions as parameters. Recall in the think condition was well above baseline and roughly the same irrespective of emotional valence. Recall of negative suppress and negative baseline items was similar, but recall of neutral suppress items was well below recall of neutral baseline items.

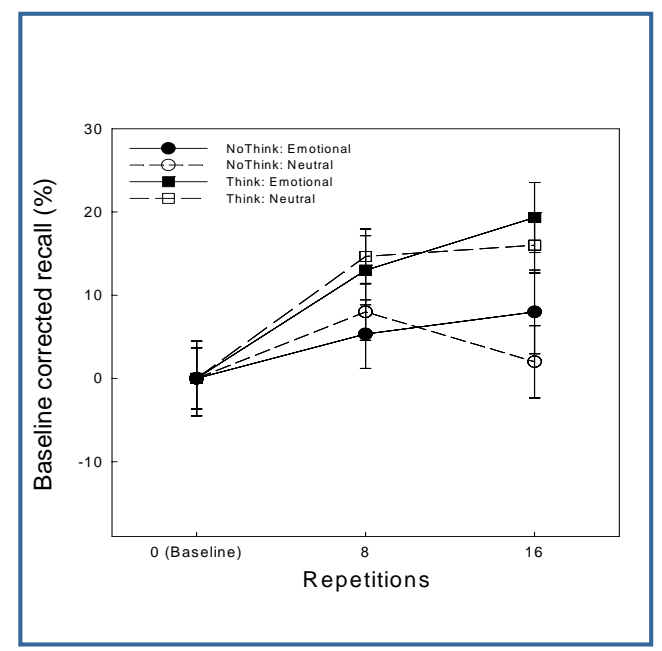

Recall data for the re-test are displayed in the right figure. Recall of repeatedly rehearsed items was well above baseline, whereas recall of neutral items previously attempted suppressed was similar to recall of emotional and baseline items.

Conclusion

Our study confirmed Anderson and Greens (2001) finding, that neutral items may be suppressed, but we found no evidence that emotional items could be suppressed below baseline, as evidenced by the results from both the immediate and delayed test. We suggest that this was the case because the emotional items were elaborated upon and encoded as more salient in memory compared to the neutral items.

However, some studies (Depue et al., 2006; 2007) have found that emotional stimuli may be suppressed. The conflicting results may stem from a difference in experimental design. Depue et al. used a blocked design in which the impact of the emotional stimuli may have leveled off after a few blocks with the result that emotional and neutral stimuli were processed in a similar way (see Marx et al., 2008).

Delayed recall of neutral to-be-suppressed targets was not inferior to recall of neutral baseline targets. We suggest that suppression in the TNT paradigm is a transient phenomenon, and that successfully suppressed material recover due to a gradual release of inhibitory processes that were instantiated in the first place to keep particular targets out of mind. 